\title{
High altitude, hyper-arid soils of the Central-Andes harbor mega-diverse communities of actinobacteria
}

\author{
Alan T. Bull ${ }^{1}$ Hamidah Idris ${ }^{2} \cdot$ Roy Sanderson $^{2}$ (D) Juan Asenjo ${ }^{3} \cdot$ \\ Barbara Andrews ${ }^{3} \cdot$ Michael Goodfellow ${ }^{2}$
}

Received: 4 September 2017 / Accepted: 24 October 2017 / Published online: 3 November 2017

(c) The Author(s) 2017. This article is an open access publication

\begin{abstract}
The data reported in this paper are among the first relating to the microbiology of hyper-arid, very high altitude deserts and they provide base line information on the structure of actinobacterial communities. The high mountain Cerro Chajnantor landscape of the Central Andes in northern Chile is exposed to the world's most intense levels of solar radiation and its impoverished soils are severely desiccated. The purpose of this research was to define the actinobacterial community structures in soils at altitudes ranging from 3000 to $5000 \mathrm{~m}$ above sea level. Pyrosequencing surveys have revealed an extraordinary degree of microbial dark matter at these elevations that includes novel candidate actinobacterial classes, orders and families. Ultraviolet-B irradiance and a range of edaphic factors were found to be highly significant in determining community compositions at family and genus levels of diversity.
\end{abstract}

Communicated by A. Oren.

Electronic supplementary material The online version of this article (https://doi.org/10.1007/s00792-017-0976-5) contains supplementary material, which is available to authorized users.

Roy Sanderson

roy.sanderson@ncl.ac.uk

Alan T. Bull

A.T.Bull@kent.ac.uk

1 School of Biosciences, University of Kent, Canterbury, Kent CT2 7NJ, UK

2 School of Biology, Ridley Building, Newcastle University, Newcastle upon Tyne NE1 7RU, UK

3 Department of Chemical Engineering and Biotechnology, Centre for Biotechnology and Bioengineering (CeBiB), University of Chile, Beauchef 851, Santiago, Chile
Keywords High altitude $\cdot$ Hyper-arid soils · Actinobacteria $\cdot \beta$-Diversity $\cdot$ Microbial dark matter

\section{Introduction}

The attraction of extreme environments for microbiologists has risen appreciably in recent years following recognition that the environmental limits to life on Earth are defined by the presence of microorganisms, the term environmental limits describing the outermost boundaries of the known physico-chemical world (Horikoshi et al. 2011). Reasons for studying the extremobiosphere (Bull 2011) in this context are several and encompass fundamental science and the hypothesis that they are likely to contain novel microorganisms that express new chemistry and metabolisms hence driving innovative biotechnology.

The most intensely researched habitats in the extremobiosphere have been hyper-thermal, deep sea and cryosphere and only more recently has attention turned to those exposed to long term and intense aridity that characterize deserts. Non-polar deserts constitute about one-third of the Earth's landmass and our own research has been based in one of the world's two coastal deserts, namely the Atacama Desert of northern Chile (Bull and Asenjo 2013; Bull et al. 2016). This desert is acknowledged to be the most arid on Earth and its aridity is maintained by a 'rain shadow' effect resulting from its enclosure between the Andean Cordillera and the Coastal Mountains. Hyper-arid and extreme hyper-arid environments (sensu Houston 2006) are characteristic of the desert's core region but the Atacama also comprises salars, Altiplano and high altitude mountain plateaus, and volcanoes.

Reports of the microbiology of high altitude mountain soils worldwide are sparse and little information is available relating to those of the Central-Andes. However, several 
investigations of high altitude wetlands (ponds, saline lakes, salterns and peatlands) at altitudes between ca. 3700 and $6500 \mathrm{~m}$ above sea level have been made in the Chilean and Bolivian Altiplano and the Central-Andes of Argentina. One such initiative, the multidisciplinary High-Lakes Project, has as a primary objective the assessment of increased environmental stress on the biological disturbance of high altitude lakes during the periods of rapid climate change as an analogy to early Mars (Cabrol et al. 2009). Other studies have started to chart the diversity and novelty of particular microbial groups (archaea and bacteria, Dorador et al. 2010, 2013; Aszalós et al. 2016; diatoms, Blanco et al. 2013), to explore polyextremophily (Albarracin et al. 2015) and diurnal community dynamics in response to solar radiation (Molina et al. 2016) in these elevated wetland habitats.

The most intensive investigations of barren high altitude soils per se have been made by Steve Schmidt and his group, several of which have been sited on the highest volcanoes in the Atacama region (Lynch et al. 2012) which they consider to be "arguably the most extreme soil ecosytems on Earth" based on extreme temperature cycling, aridity, low atmospheric pressure and extreme solar radiation. In a series of papers Schmidt and his colleagues reported pioneering studies of the microbiota of fumarolic and non-fumarolic soils of the Socompa and Llullaillaco volcanoes between 5500 and $6330 \mathrm{~m}$ a.s.l. These communities are among the simplest found in terrestrial ecosystems and appear to be sustained by the activities of facultative chemoautotrophic bacteria (Costello et al. 2009; Lynch et al. 2012).

This paper reports the latest results of our on-going research on desert soil actinobacteria, the research landscape being the Chilean high mountain desert, Cerro Chajnantor (5640 m a.s.1.), which lies east of San Pedro de Atacama. This landscape is subject to a combination of extreme environmental conditions including the world's highest levels of surface UV radiation (Cordero et al. 2014). In this study metagenomic profiles of actinobacterial communities in these very high altitude desert soils were compared with those of low altitude desert soils, previously defined by
(Idris et al. 2017a), to test the hypothesis of microbiome taxonomic distinctiveness of Atacama Desert soils. In addition, attempts have been made to identify the principal determinants of actinobacterial community structure as a function of soil environment and climate characteristics.

\section{Materials and methods}

\section{Research landscape and sample collection}

Replicate samples (4) were collected aseptically in October 2012 and stored as described previously (Idris et al. 2017a). Surface and subsurface samples were taken at three altitudes on the Cerro Chajnantor (Table 1). At the two lower altitudes the ecosystems contained sparse vegetation largely comprising Andean grasses, xerophytes and columnar cacti, particularly Echinopsis atacamensis (Fig. 1). The highest sampling site (5041 $\mathrm{m}$ a.s.l.) lay within the periglacial zone as defined by Schmidt et al. (2012).

The Cerro Chajnantor is a lava dome and part of a large volcanic complex formed on top of a pyroclastic shield. It is one of several volcanic peaks that border the Chajnantor Plateau (ca. $5000 \mathrm{~m}$ a.s.l.), a region of the southern Altiplano that is the location of a group of astronomical observatories including the Atacama Large Millimeter Array (ALMA). ALMA is operated as an international partnership of which the European Southern Observatory (ESO) is a member. Permission to collect samples was given by the ESO and in accordance with its requirements health checks were made at the ALMA Operations Support Facility (2900 m a.s.l.) prior to sampling at higher altitudes.

\section{Soil analyses and climate data}

Soil measurements and elemental analyses were made at the Geochemistry Laboratory, University of Chile, Santiago; and UV irradiance data were collected at the Chajnantor Plateau in February-March 2015 by Dr. Raul Cordero

Table 1 Cerro Chajnantor research site data

\begin{tabular}{|c|c|c|c|c|c|c|c|c|}
\hline \multirow{2}{*}{$\begin{array}{l}\text { Location/sampling } \\
\text { depth }(\mathrm{cm})\end{array}$} & \multirow{2}{*}{ Latitude ${ }^{\circ} \mathrm{S}$} & \multirow[t]{2}{*}{ Longitude ${ }^{\circ} \mathrm{W}$} & \multirow{2}{*}{$\begin{array}{l}\text { Altitude (m } \\
\text { a.s.1.) }\end{array}$} & \multirow{2}{*}{$\begin{array}{l}\text { Atmospheric } \\
\text { pressure (bar) }\end{array}$} & \multirow[t]{2}{*}{ Air temp $\left({ }^{\circ} \mathrm{C}\right)$} & \multicolumn{2}{|c|}{ Radiation $\left(\mathrm{kJ} / \mathrm{m}^{2 /}\right.$ day) } & \multirow[t]{2}{*}{ Vegetation } \\
\hline & & & & & & $U V-A^{a}$ & $U V-B^{b}$ & \\
\hline ALMA 1 (2) & $23^{\circ} 04^{\prime} 39^{\prime \prime}$ & $67^{\circ} 57^{\prime} 43^{\prime \prime}$ & 3018 & 1015 & 25.0 & 1545 & 46.0 & Present \\
\hline ALMA 2 (30) & $23^{\circ} 04^{\prime} 39^{\prime \prime}$ & $67^{\circ} 57^{\prime} 43^{\prime \prime}$ & 3018 & 1015 & 25.0 & & & \\
\hline ALMA 3 (2) & $23^{\circ} 03^{\prime} 31^{\prime \prime}$ & $67^{\circ} 52^{\prime} 27^{\prime \prime}$ & 4000 & 1015 & 27.2 & 1579 & 49.0 & Present \\
\hline ALMA 4 (30) & $23^{\circ} 03^{\prime} 31^{\prime \prime}$ & $67^{\circ} 52^{\prime} 27^{\prime \prime}$ & 4000 & 1015 & 27.2 & & & \\
\hline ALMA 5 (2) & $23^{\circ} 00^{\prime} 49^{\prime \prime}$ & $67^{\circ} 45^{\prime} 31^{\prime \prime}$ & 5041 & 1015 & 15.6 & 1621 & 51.2 & Absent \\
\hline ALMA 6 (30) & $23^{\circ} 00^{\prime} 49^{\prime \prime}$ & $67^{\circ} 45^{\prime} 31^{\prime \prime}$ & 5041 & 1015 & 15.6 & & & \\
\hline
\end{tabular}

a $315-400 \mathrm{~nm}$

b $280-315 \mathrm{~nm}$ 
Fig. 1 Sampling locations on the Cerro Chajnantor. a ALMA

1 site at $3018 \mathrm{~m}$ a.s.l. with Salar de Atacama in the background. b ALMA 3 site at $4000 \mathrm{~m}$ a.s. 1 . showing scattered colonization by Echinopsis atacamensis. c Landscape between ALMA sites 3 and 5 with Licancabur Volcano in the background. $\mathbf{d}$ ALMA 5 site at $5041 \mathrm{~m}$ a.s.l. on the Chajnantor Plateau with the peak of Cerro Chajnantor in the background
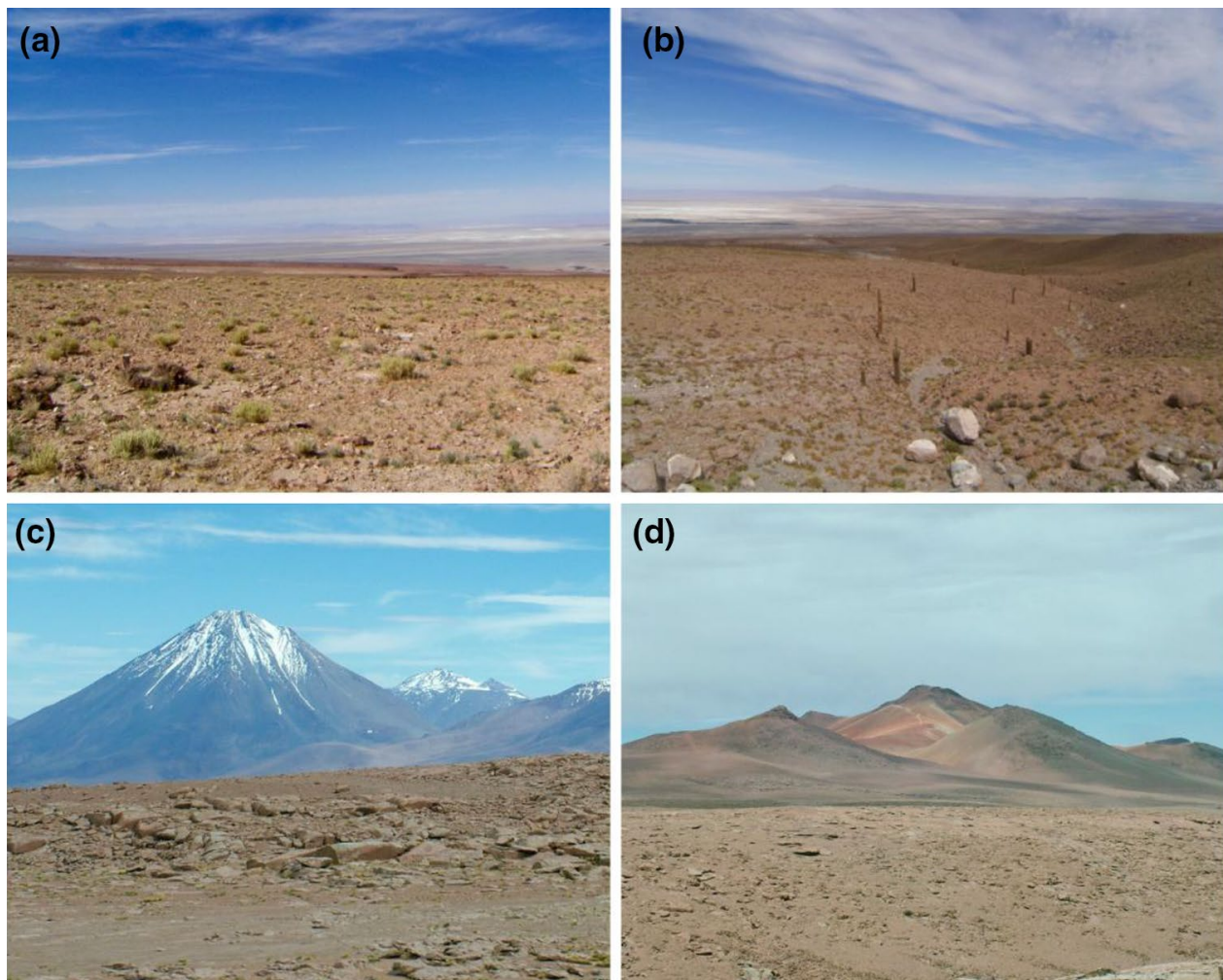

(Department of Physics, University of Chile, Santiago). Latitude, longitude, elevation and other climatic data were collected during sampling using a handheld multi-function altimeter (AltiTech2, Highgear Inc., Durham, NC, USA).

\section{DNA extraction, PCR amplification and pyrosequencing}

All of the procedures used were those described by us previously (Idris et al. 2017a).

\section{Bioinformatic and multivariate statistical analyses}

A total of 12,388 valid pyrosequence reads were processed using CLCommunity v3.30 software at Chunlab Inc. (http:// www.chunlab.com; Seoul National University, Korea) and taxonomic assignments to actinobacterial family and genus ranks were made using the EzBioCloud (Yoon et al. 2017) database (see Idris et al. 2017a for details of these procedures).

Alpha-diversity analyses including rarefaction, rankabundance, Shannon diversities and Chaol richness estimations were calculated using CLCommunity software. Operational Taxonomic Unit tables (OTU) were saved in comma delimited (.csv) format prior to further analysis. Identified generic phylotypes were checked manually from OTU tables and analyzed to determine their composition in each of the sample sites. Venn diagrams were constructed using the Mothur version 1.37.4 program (http://www. mothur.org/; Schloss et al. 2009). Beta-diversity analyses of actinobacterial communities were made using unconstrained principal components (PCA) and constrained redundancy (RDA) analyses using the "vegan" (Oksanen et al. 2013) package to explore the effects of environmental variables on community composition. The ANOVA permutation test (Ter Braak 1986) was used to assess the significance of such variables on community structure.

\section{Results}

\section{Site characteristics}

The texture of sampled material varied from coarse soil, to gravel, regolith, and rock fragments the moisture contents of which were zero with the exception of the subsurface at the highest altitude (Table 2), the latter reflecting its periglacial nature. Ultraviolet irradiance levels are quoted as doses $\left(\mathrm{kJ} / \mathrm{m}^{2}\right)$ and computed by integrating through time to provide a daily dosage. UV-A and UV-B irradiance increased by 4.9 and $11.3 \%$ over the altitude range sampled (Table 1), values agreeing closely with those reported in a major survey of solar spectral conditions in the Atacama Desert, including the Chajnantor Plateau (Cordero et al. 2016). On the basis of redox 
Table 2 Physico-chemical characteristics of Cerro Chajnantor soils

\begin{tabular}{|c|c|c|c|c|c|c|c|c|c|c|c|}
\hline \multirow[t]{2}{*}{ Location } & \multirow[t]{2}{*}{$\mathrm{pH}$} & \multirow{2}{*}{$\begin{array}{l}\text { Redox poten- } \\
\text { tial }(\mathrm{mV})\end{array}$} & \multirow{2}{*}{$\begin{array}{l}\text { Conductivity } \\
(\mu \mathrm{S} / \mathrm{cm})\end{array}$} & \multirow[t]{2}{*}{ Moisture (\%) } & \multirow{2}{*}{$\begin{array}{l}\text { Total organic } \\
\text { content }(\%)\end{array}$} & \multirow[t]{2}{*}{ Texture } & \multicolumn{5}{|c|}{ Elements (\%) } \\
\hline & & & & & & & $\mathrm{Ca}$ & $\mathrm{Mg}$ & $\mathrm{Na}$ & $\mathrm{K}$ & $\mathrm{Fe}$ \\
\hline ALMA 1 & 7.08 & 360 & 215 & 0 & 1.67 & Coarse mineral soil & 3.9 & 1.3 & 2.7 & 1.8 & 4.8 \\
\hline ALMA 2 & 7.63 & 336 & 326 & 0 & 3.67 & Coarse mineral soil & 3.2 & 1.3 & 2.0 & 2.2 & 3.9 \\
\hline ALMA 3 & 6.55 & 360 & 239 & 0 & 3.33 & Regolith & 3.2 & 1.3 & 2.6 & 1.9 & 4.5 \\
\hline ALMA 4 & 6.70 & 335 & 108 & 0 & 3.67 & Gravel & 3.0 & 1.5 & 2.4 & 1.8 & 4.5 \\
\hline ALMA 5 & 6.73 & 369 & 54 & 0 & 2.00 & Regolith/rock & 3.6 & 1.4 & 2.4 & 2.7 & 4.1 \\
\hline ALMA 6 & 6.30 & 365 & 44 & 4.67 & 0.67 & Gravel & 3.5 & 1.4 & 2.4 & 2.7 & 4.1 \\
\hline
\end{tabular}

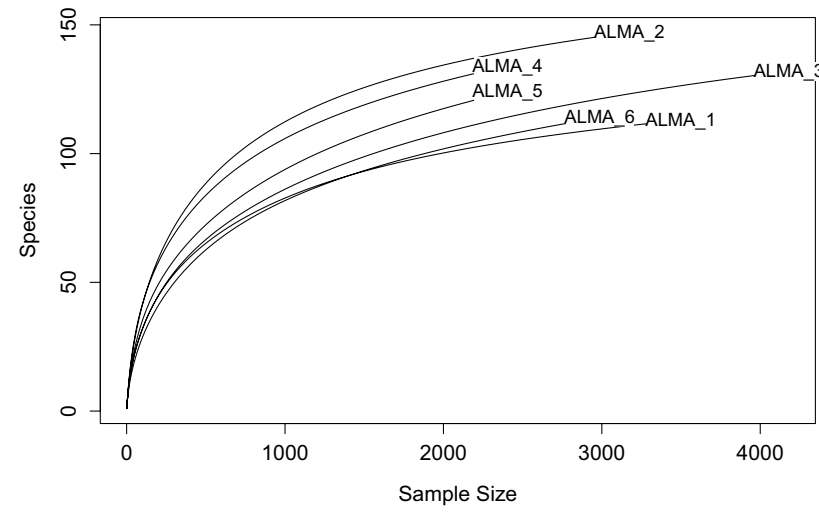

Fig. 2 Rarefaction analyses of ALMA OTUs at 94.5-97.0\% phylogenetic similarity showing the relation between the increase in generic richness and the number of randomly sampled sequences

potential values (Husson 2013) all of the soils could be designated as dry and moderately oxidizing and showed little inter-site variation. The nature of possible oxidants in these soils has not been investigated but in part may be related to very high UV radiation flux. Soil conductivity has a strong correlation with salinity and also correlates with soil texture and particle size, an effect that was particularly noticeable in the very low values recorded at the $5041 \mathrm{~m}$ sites. All of the soils contained relatively high concentrations of $\mathrm{Ca}$ and $\mathrm{Na}$ that are indicative of high salt contents, a condition of Atacama Desert soils regarded as indicative of persistent aridity (Crits-Christoph et al. 2013) (Table 2).

\section{Community coverage and composition}

Our pyrosequencing programme produced an average of 2956 sequence reads per soil sample most of which had reads of > 1000; this average read number is smaller than that we obtained for low altitude Atacama soils using identical procedures (Idris et al. 2017a) but compares very favorably with low altitude Atacama soil surveys reported earlier by Crits-Christoph et al. (2013).
Table 3 Observed generic OTUs and $\alpha$-diversity indices

\begin{tabular}{llllll}
\hline Location & $\begin{array}{l}\text { Good's } \\
\text { library cov- } \\
\text { erage (\%) }\end{array}$ & $\begin{array}{l}\text { Observed } \\
\text { OTUs }\end{array}$ & Chao 1 & $\begin{array}{l}\text { Shannon } \\
\text { index }(H)\end{array}$ & $\begin{array}{l}\text { Simpson } \\
\text { diversity } \\
(1 / D)\end{array}$ \\
\hline ALMA 1 & 97 & 158 & 195 & 4.03 & 33.3 \\
ALMA 2 & 95 & 354 & 484 & 5.15 & 100 \\
ALMA 3 & 98 & 211 & 256 & 4.41 & 50 \\
ALMA 4 & 95 & 233 & 373 & 4.59 & 50 \\
ALMA 5 & 95 & 224 & 331 & 4.52 & 50 \\
ALMA 6 & 97 & 207 & 257 & 4.12 & 33.3 \\
\hline
\end{tabular}

Inspection of rarefaction curves (Fig. 2) shows that they tend to asymptotes for each of the soils thereby arguing for high sequence coverage, a conclusion strongly supported by reference to Good's coverage metric (Table 3). With the exception of the subsurface gravel from $3018 \mathrm{~m}$ a.s.1. (ALMA 2), the Shannon and Simpson Indices do not provide any evidence for dramatic changes in generic diversity as a function of altitude, although generic richness in terms of observed OTUs and Chao estimates showed greater variability (Table 3 ). The reciprocal of Simpson's index is sensitive to the level of dominance in microbial communities but only in the $3018 \mathrm{~m}$ surface and the $5041 \mathrm{~m}$ subsurface soils were dominant profiles evident $(1 / D<50$; Zhou et al. 2002) while the remainder possessed uniform community profiles.

When sequences were assigned to OTUs, 55 families and 234 genera of actinobacteria were defined; of these 33 and $41 \%$, respectively, could not be assigned to valid named taxa and hence are considered to be putatively novel candidate families and genera (Supplementary Material, Tables 1 and 2). A significant proportion (20\%) of identified families could be assigned to deep lineage actinobacteria belonging to the class Acidimicrobia (Norris 2012), a majority of which appear to be novel. As in our previous report (Idris et al. 2017a) we have used a rank-abundance threshold of $0.1 \%$ to define a rare actinobacteria biosphere (sensu Lynch and Neufeld 2015); using this criterion $40 \%$ 
of detected families and $65 \%$ of detected genera belong to the rare microbiosphere.

\section{Taxonomic diversity and variation between sites and depths}

The extraordinary novelty among high taxonomic rank actinobacteria occurring in low altitude Atacama Desert ecosystems (Idris et al. 2017a) was found to be comparable at the Cerro Chajnantor sites, with phylotypes representing 2 putatively new classes, 4 new orders and 18 new families in the phylum Actinobacteria being recorded (cumulative site data, Supplementary Material, Table 1). The large majority of OTUs were identified as members of the orders Frankiales (34\%), Micrococcales (9\%), Acidimicrobiales (7\%), Propionibacteriales (7\%) and Pseudonocardiales (5\%); sequence reads corresponding to OTUs of a putative new order FJ478799_o accounted for 2.5\% of the Chajnantor community.

The most frequently detected genera comprizing $64 \%$ of the actinobacterial phylotypes were in order of abundance: HQ910322_g, Blastococcus, Arthrobacter, Modestobacter, Geodermatophilus, Mycobacterium, HQ674860_g, JF266448_g, Friedmanniella, FJ479147_g, Pseudonocardia, Nocardioides, Jatrophihabitans, Terrabacter and Sporichthya (Supplementary Material, Table 2). The abundance of certain genera (Blastococcus, Modestobacter, Geodermatophilus, Friedmanniella) was clearly related to soil depth and to altitude (Fig. 3) and probably reflects an ability to withstand irradiation stress; in all cases abundance fell progressively with altitude. All of these organisms are contained in the Terrabacteria group of prokaryotes (Battistuzzi and Hedges 2008) that is distinctive of hyper-arid deserts and notable for containing organisms selected to withstand environmental stress including irradiations and desiccation. Gtari et al. (2012) studied stress phenomena in selected strains of these actinobacterial clades and found high tolerance to UV radiation especially in melanized G. obscurus and M. multiseptatus but less so in $B$. saxobsidens that produces orange pigmentation. Protective UV-absorbing mycosporine-like amino acids have recently been reported in a member of the genus Pseudonocardia (Miyamoto et al. 2014), another of the most abundant actinobacterial taxa in Chajnantor soils. Also of note is that most of the identified high abundance generic phylotypes are known to produce coccoid cells and in some cases spores, morphological features that Gtari et al. (2012) opine may reflect adaptations to arid habitats.

The proportion of identified co-occurring generic phylotypes in surface and subsurface soils at each altitude was approximately $50 \%$; however, greater differentiation of actinobacterial communities was evident in between-surface and between-subsurface communities where co-occurrence was only 30\% (Supplementary Material, Figure 1). Sub-surface communities tended to be more taxonomically rich but the differences were small $(<10 \%)$. Previously unreported phylotypes were detected in all of the soils.
Fig. 3 Effects of altitude and soil depth on the abundance of the most dominant genera; sites 1 and 2 at $3018 \mathrm{~m}$ a.s.l.; sites 3 and 4 at $4000 \mathrm{~m}$ a.s.l.; sites 5 and 6 at $5041 \mathrm{~m}$ a.s.1.. Samples at sites 1, 3, 5 taken at $2 \mathrm{~cm}$ depth; sites 2, 4 and 6 at $30 \mathrm{~cm}$ depth

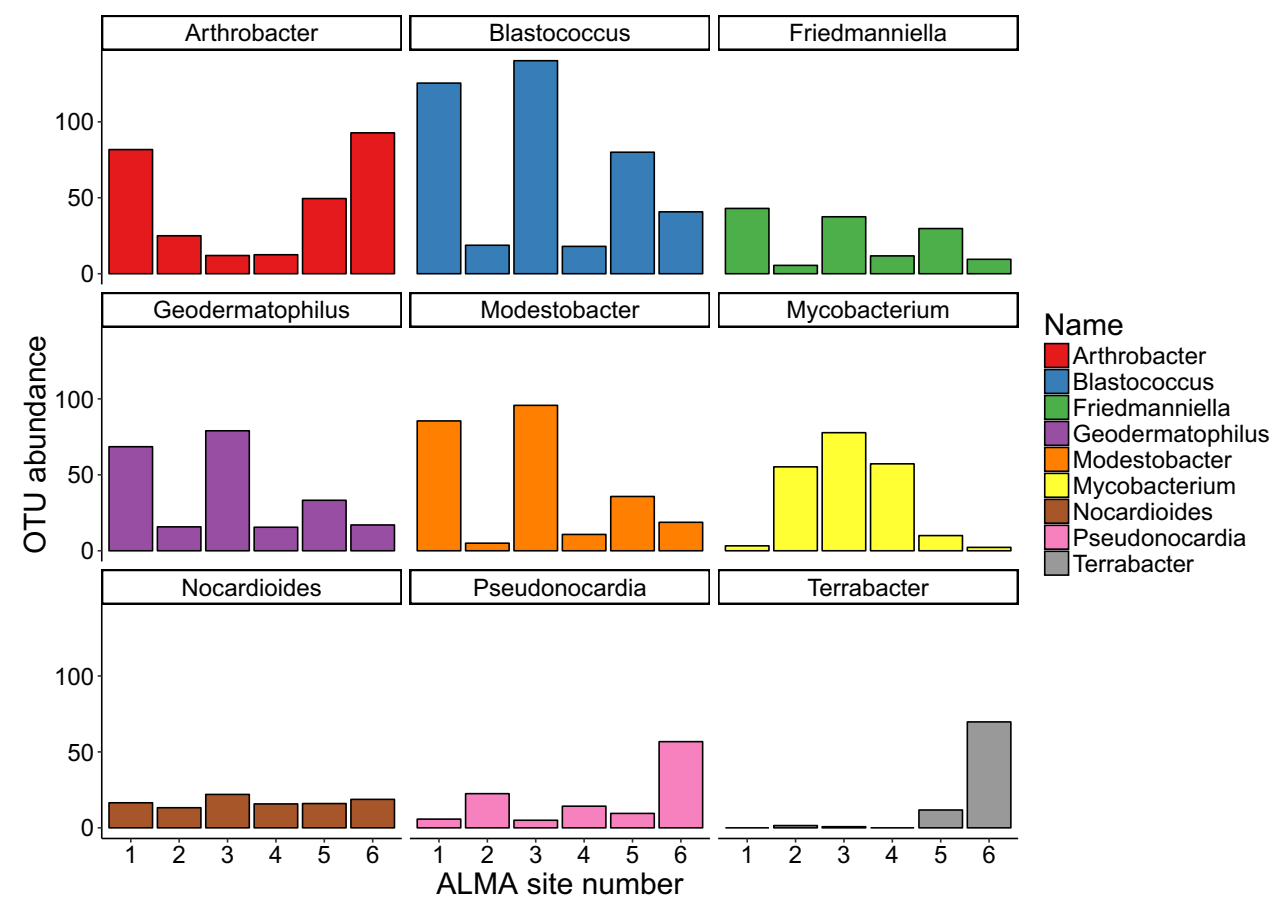


The total variation explained by the first two principal component axes was relatively low at $26 \%$, but nevertheless distinct patterns could be observed. Two broad community groups can be defined (Fig. 4a): Group 1 comprised those communities at ALMA sites 1, 5 and 6 (upper left of the PCA plot), while the more diffuse Group 2 comprised communities at ALMA sites 2, 3 and 4. Substantial within-site variation in community composition was evident at some of the sites (ALMA 2, subsurface, $3018 \mathrm{~m}$, and ALMA 3, surface, $4000 \mathrm{~m}$ ) as indicated by the larger spread of polygons in PCA ordination space (Fig. 4a); other sites had relatively little variation in composition between replicate samples. The PCA notably differentiates surface and subsurface communities at the lower elevations. The overall trends are clearly discerned in the box plot presentation of the data that summaries changes in PCA axis 1 scores with altitude and sampling depth (Fig. 4b); as altitude increased there was a gradual convergence in community composition between the surface and subsurface samples, as well as a decline in within-community variability of taxonomic composition.

At the lowest altitude $(3018 \mathrm{~m})$, there are major differences in the community composition depending on depth, and also more variability between samples in the subsurface (ALMA 2) than surface (ALMA 1). The surface samples, that are subject to high levels of UV radiation, did not appear to change much in community composition as altitude increased from 3018 to $5041 \mathrm{~m}$, as their PC Axis 1 score remained constant, with a similar amount of variation around the mean. In contrast, the community composition of subsurface samples (ALMA 2, ALMA 4 and ALMA 6) changed dramatically with altitude, with the PC Axis 1 score gradually converging to that of the surface samples by $5041 \mathrm{~m}$, indicating that at the highest altitudes surface and subsurface samples have similar actinobacterial community composition. Moreover, individual subsurface samples became less variable with increasing altitude suggestive of increased stresses at high altitude even within the subsurface environment.
Fig. 4 a Principal component analysis of surface and subsurface communities. PC1 and PC2 contributed 15 and $11 \%$ of the variance, respectively, $\mathbf{b}$ box plot summarising the status of actinobacterial communities with regard to altitude and sample depth. Interquartile ranges are represented by box outlines, median values by midlines, and maximum and minimum values by whiskers (a)

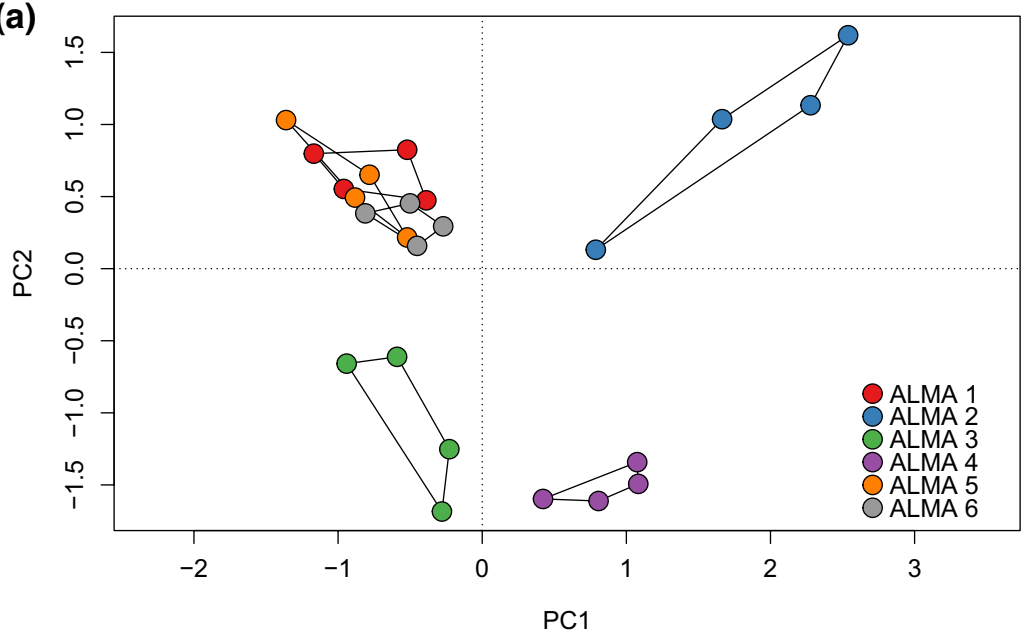

(b)

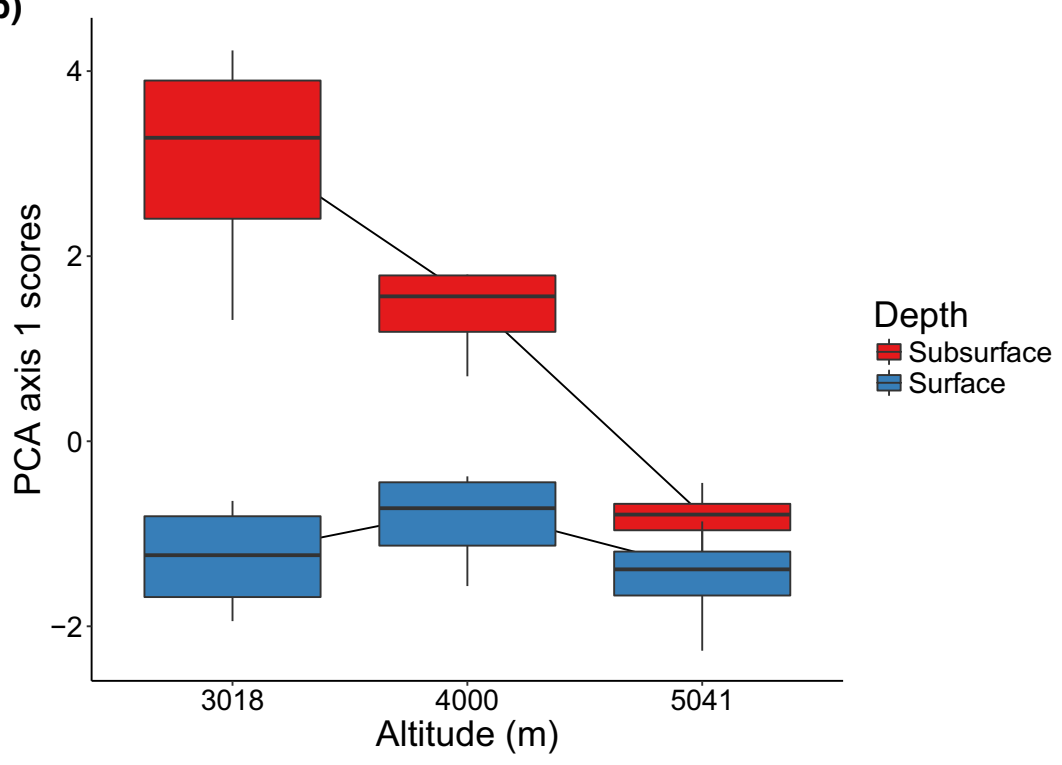




\section{Beta-diversity}

The two actinobacterial community groups suggested by principal components analysis were confirmed by the construction of a double annotated hierarchical heat map based upon the 138 validated generic phylotypes (Supplementary Material, Figure 2) and, moreover, the majority of the previously most frequently detected and abundant phylotypes (Supplementary Material, Table 1) were assignable to the Group 1 communities. The heat map Group 1 communities were dominated by members of the family Geodermatophilaceae and the genera Friedmanniella, Sporichthya and Terrabacter, while other phylotypes were co-dominant in the two community groups, notably the genera Jatrophihabitans, Nocardioides and Pseudonocardia. These dominant phylotypes were most conspicuous in surface soil sites of the Group 1 communities, i.e. at 3018 and $5041 \mathrm{~m}$ a.s.l., again suggesting an enhanced tolerance to radiation stresses.

Relationships between community composition and environmental variables were examined using redundancy analysis (RDA), the results of which are summarized in Fig. 5. In the RDA ordination diagram, the length of the arrow for each constraining variable indicates the magnitude of its effect, arrows pointing in the same direction are positively correlated with each other; arrows at $90^{\circ}$ to each other are uncorrelated, and arrows at $180^{\circ}$ are negatively correlated.

Samples positively associated with constraining variables are located near the arrow heads. Of the variables tested, soil redox potential and organic matter are the most important drivers of community composition (although they are negatively correlated with each other) and are positively correlated with altitude and soil conductivity, respectively. Soil pH appeared to be correlated only with the variable subsurface soil samples at $3018 \mathrm{~m}$ (see Fig. 5) which was the most alkaline of all those tested. Results of RDA permutation ANOVA tests showed decisively that all of the environmental variables were highly significant in determining actinobacterial community compositions in the Cerro Chajnantor landscape (Table 4). Large $F$ ratios indicate significant results and $p$ values $<0.05$ refute the null hypothesis that community composition is unrelated to environmental variables.

The strong positive correlation between redox potential and altitude and its association with the Group 1 communities was particularly noteworthy given the fact that Atacama Desert soils have long been known to possess strong oxidizing properties (Navarro-Gonzalez et al. 2003) although the nature of the oxidant(s) has remained elusive. Recently, however, Georgiou et al. (2015) have demonstrated that photogenerated metal superoxides and peroxides accumulate in top soils in the Yungay location of the Atacama Desert and opine that they furnish some key elements of reactive oxygen species in desert soils. Based on data contained in Cordero et al. (2016) we estimate that radiation levels imposed on Cerro Chajnantor soils to be approximately $17 \%$ greater than those at the Yungay sites. Subsequently we have used redundancy analysis to examine the relationship of community composition with UV-B irradiance (Fig. 6) and found it to be singularly correlated at the highest altitude.

Table 4 RDA permutation test of Cerro Chajnantor environmental variables

\begin{tabular}{lll}
\hline Environmental variable & $F$ ratio & $p$ value \\
\hline Altitude & 1.6716 & 0.008 \\
UV-B dosage & 2.1011 & 0.014 \\
$\mathrm{pH}$ & 3.0075 & 0.001 \\
Redox & 2.2539 & 0.001 \\
Conductivity & 1.6550 & 0.004 \\
Organic matter & 1.6143 & 0.018 \\
\hline
\end{tabular}

$F$ ratios based on 999 permutations
Fig. 5 Redundancy analysis biplot showing relationships between identified actinobacterial communities and environmental variables. Surface samples are ALMA 1, 3 and 5; subsurface ALMA 2, 4 and 6. RDA1 explains $12 \%$ and RDA2 $9 \%$ of the variation

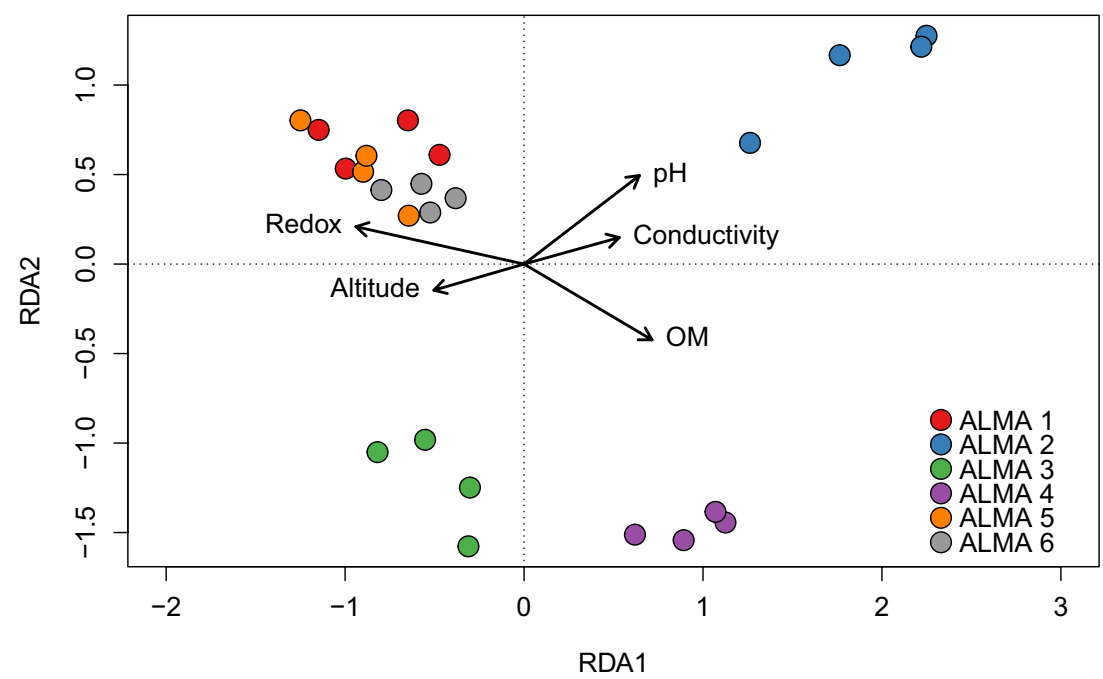


Fig. 6 Redundancy analysis biplot showing relationships between identified actinobacterial communities and UV-B irradiance. ALMA $1=3018 \mathrm{~m}$; ALMA $3=4000 \mathrm{~m}$; ALMA $5=5041 \mathrm{~m}$. RDA1 explains $23 \%$ and RDA $29 \%$ of the variation

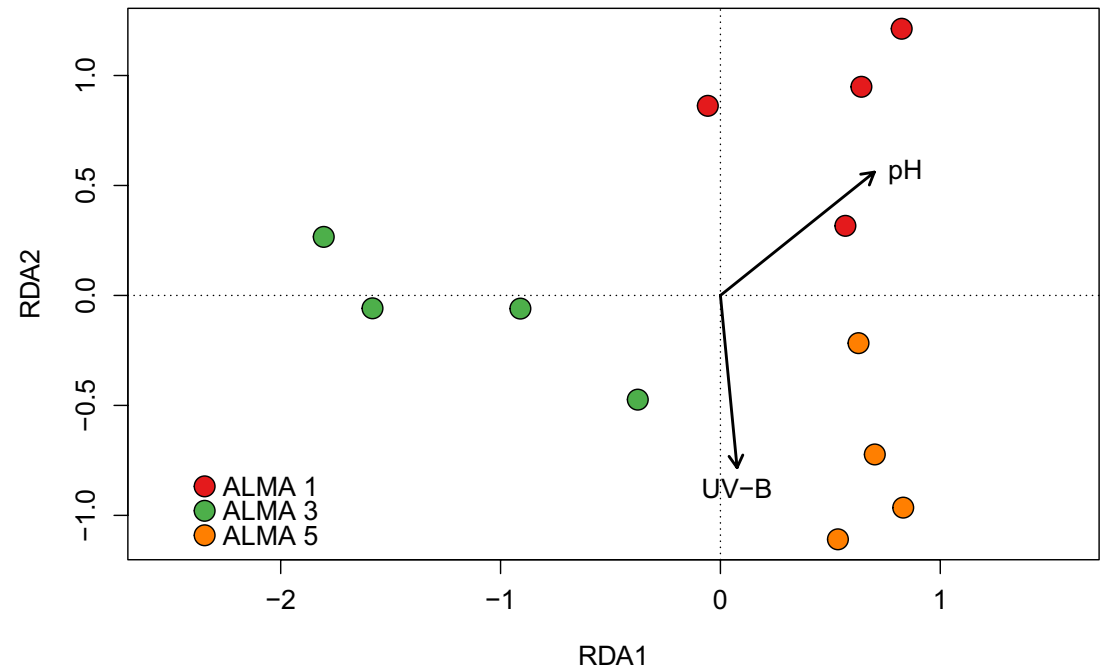

When actinobacterial family distributions are superimposed on the RDA biplot (data not shown), those most strongly correlated with UV-B irradiation were found to be the Corynebacteriaceae, Microbacteriaceae and Pseudonocardiaceae, and to a lesser degree the Nocardiaceae and Sporichthyaceae. Perhaps surprisingly the Geodermatophilaceae whose phylotypes dominated the Group 1 communities were negatively correlated with UV irradiation. Reports on UV resistance of desert bacteria tend to be rather anecdotal and little information is available on resistance mechanisms per se. Early studies showed that UV resistant actinobacteria often dominated desert communities such as rock varnish (Kuhlman et al. 2005) and that Geodermatophilus isolates in particular were resistant to high radiation dosage (Rainey et al. 2005). The latter authors remarked that "The recovery of large numbers of extremely ionizing-radiation-resistant bacteria from an arid soil and not from a non-arid soil provides further ecological support for the hypothesis that the ionizing-radiation resistance phenotype is a consequence of the evolution of other DNA repair systems that protect cells against commonly encountered environmental stressors, such as desiccation". Recently the UV-C resistance of bacteria isolated from the Atacama and Sonoran Deserts was found to be greater than or comparable to that of Deinococcus radiodurans (Paulino-Lima et al. 2016). Significantly all resistant isolates had intracellular manganese/iron ratios very much higher than those of sensitive ones although resistance was not correlated with the availability of $\mathrm{Mn}$ or soil Fe/Mn ratios. Despite the fact that the soils studied by Paulino-Lima et al. (2016) and those of the Cerro Chajnantor sites contain only low concentrations of Mn, mechanisms have been identified for its uptake and accumulation from low Mn environments (Sun et al. 2010). It remains to be investigated whether or not high altitude actinobacteria reported in this paper resist extreme UV stress by means of manganese transport and regulation systems to control recovery from radiation injury as proposed by Daly et al. (2004).

\section{Discussion}

Previously we postulated the existence of a core actinobacterial microbiome in low altitude Atacama soils that may play a key role in ecosystem function (Idris et al. 2017a); the composition of this core microbiome accords very closely with that defined for the Cerro Chajnantor sites in which phylotypes of the following families were dominant (percentage dominance in brackets): Geodermatophilaceae (19.6), HQ910322_f (9.5), Acidimicrobiaceae (8.3), Pseudonocardiaceae (7.9), Micrococcaceae (5.9), Nocardioidaceae (5.8), Microbacteriaceae (5.5), and Micromonosporaceae (5.3). The one anomaly was Candidatus FJ479147_f that was the most abundant family (18.4\% total phylotypes) in low altitude Atacama soils but had relatively low abundance in the Chajnantor $(2.6 \%)$.

Very few microbiological studies have been made of nutrient poor, high altitude desert soils and of the biological and edaphic pressures that select for community structures. We have sought to reveal adaptive traits that are plausibly associated with the fitness of actinobacteria to high altitude Atacama Desert soils to promote what Lynch et al. (2014) have reasoned "the testing of hypotheses for future culture-based experiments". These authors have pointed to the Gram-positive cell wall architecture and widespread capacity to sporulate as possible early adaptations to arid environments and it is notable that a high proportion $(30 \%)$ of the most dominant actinobacterial phylotypes identified in Cerro Chajnantor soils are amycelial and/or known spore producers. Although UV-B accounts for only a small fraction of total solar irradiation, dosage levels on the Cerro Chajnantor are the world's highest recorded and its effect is greatly enhanced as a consequence of extremely low values of precipitable water $(<1 \mathrm{~mm})$, total ozone column and atmospheric aerosols, and high altitude (Cordero et al. 2014, 2016). Figure 6 pointedly indicates that the highest altitude 
surface soil (ALMA 5) correlated most strongly with UV-B dosage (near the tip of the UV arrow) whilst the ALMA 1 soil (lowest altitude) is at the top of the graph with lowest UV levels. Whereas our finding of a high abundance of Geodermatophilaceae phylotypes at this highest altitude site accords with the ability to isolate members of this family from other desert top soils and from exposed stone surfaces (Busarakam et al. 2016; Gtari et al. 2012; Urzì et al. 2001), redundancy analysis failed to establish a correlation with UV-B irradiance. It is feasible, therefore, that the abundance of these bacteria in Cerro Chajnantor soils may be correlated with their resistance to other stress factors that give rise to reactive oxygen species (ROS) such as severe desiccation and soil redox potentials, a proposition that is supported by the highly significant results of redundancy analyses (Table 4). The redundancy analysis reveals a large amount of unexplained variation in our data (over $70 \%$ ). Some of this unexplained variation may be a result of unmeasured environmental variables: for example a more detailed assay of the chemical composition of the underlying geology at the six sampling sites may have provided additional explanatory variables to include in the RDA. Nevertheless, it is likely that actinobacteria in extreme desert environments are subject to considerable spatial and temporal environmental stochasticity (Caruso et al. 2011), adding to the variation in community composition.

A salient outcome of Schmidt's metagenomic surveys of Atacama and other Andean volcanic soils was the hypothesis that organic carbon could be generated by chemoautotrophic microorganisms thereby sustaining communities in such harsh, nutrient impoverished environments. Samples from the Llullaillaco Volcano $6034 \mathrm{~m}$ a.s.l. were dominated by actinobacterial phylotypes among which a Pseudonocardia species was the most abundant (Lynch et al. 2014), the genome of which encoded for $\mathrm{CO}_{2}$ fixation via the Calvin cycle and complete pathways for $\mathrm{C} 1$ compounds, hydrogen, ammonia and nitrate assimilation. The family Pseudonocardiaceae is the fourth most abundant in Cerro Chajnantor soils and in $5041 \mathrm{~m}$ samples the abundance of a Pseudonocardia phylotype reached 13\% (Supplementary Information, Table 2). The high incidence of Arthrobacter and Terrabacter phylotypes at this highest altitude site investigated ( 8 and $21 \%$ abundance, respectively; Fig. 3 ) is also notable given reports of carboxydovory in these genera (Kim et al. 2011; Lalonde and Constant 2016).

Future research priorities undoubtedly include the identity and characterization of adaptive traits in these high altitude desert communities. An immediate opportunity is afforded by the collection of isolates representing several of the dominant actinobacterial families, including the families Geodermatophilaceae and Pseudonocardiaceae, for culturebased physiology investigations and whole genome mining. To date we have whole genome sequences for two cultured members of the Geodermatophilaceae (Modestobacter caceresii, Busarakam et al., 2016, and Geodermatophilus atchiseltius sp. nov., J. F. Castro et al., unpublished data) both of which encode a high proportion of environmental stress related genes and others associated with chemolithotrophic metabolism. However, members of many of the most abundant lineages such as HQ910322_f have yet to be brought into culture or even characterized taxonomically and given the extent of the actinobacterial dark matter in the Cerro Chajnantor soils this is a major task. Future soil surveys based on a multi-omics approach in which metagenomics is complemented by metatranscriptomics and metaproteomics should enable active and dormant actinobacteria in the communities to be distinguished (Aanderud et al. 2015) and to provide insights into the functional genomics of habitat adaptation as demonstrated by Lynch et al. (2014).

\section{Conclusions}

The Atacama Desert landscape is an extraordinary repository of actinobacterial 'dark matter' with significant numbers of Candidatus taxa detected at supra-generic levels and nearly $50 \%$ of phylotypes not being assigned to validly named genera. Similarly high are our estimates of the actinobacterial rare biosphere in these high altitude environments, measuring $65 \%$ of all detected generic phylotypes.

At supra-genus rank 2 putatively new classes, 4 new orders and 18 new families in the phylum Actinobacteria have been defined and such, we opine, represents mega-diversity.

The extremely low aerosol optical depth values were recorded at high altitude sites in the Atacama Desert, particularly on the Chajnantor Plateau, (Cordero et al. 2016) has implications for habitat contamination by deserts dusts blown in either from local or distant geographic sites and is strongly indicative that the microbial communities defined in this study can be categorized as indigenous.

The actinobacterial communities found in extreme arid habitats of the Atacama Desert present unique opportunities for bioprospecting. Thus, initial screening of the Cerro Chajnantor culture collection has shown that approximately $20 \%$ of all isolates gave positive results in a Bacillus subtilis reporter strain survey, while a new species of the rare genus Lentzea, L. chajnantorensis (Idris et al. 2017b) isolated from the $5041 \mathrm{~m}$ site, produces novel diene and monoene glycosides some of which have anti-HIV integrase activities (Wichner et al. 2017).

Acknowledgements We thank ALMA Director of the ESO for permission to collect samples; the Laboratorio Geoquimica, University of Chile, Santiago for chemical analyses; Dr. Raul Cordero, Department of Physics, University of Chile, Santiago for UV data; and ChunLab, Inc., Seoul for assistance with pyrosequence analyses. 
Author contributions $\mathrm{HI}$ conducted the experiments that were conceived and planned by ATB, MG and HI; ATB, JAA and MG collected environmental samples; BAA organized soil analyses; and RS analyzed results. The manuscript was written by ATB and reviewed by all authors.

\section{Compliance with ethical standards}

Funding ATB and JAA thank the Royal Society for International Joint Project Grant JP100654, HI the Malaysian Government for a postgraduate scholarship, and MG the Leverhulme Trust for an Emeritus Fellowship.

Conflict of interest The authors declare no competing financial interests.

Open Access This article is distributed under the terms of the Creative Commons Attribution 4.0 International License (http://creativecommons.org/licenses/by/4.0/), which permits unrestricted use, distribution, and reproduction in any medium, provided you give appropriate credit to the original author(s) and the source, provide a link to the Creative Commons license, and indicate if changes were made.

\section{References}

Aanderud ZT, Jones SE, Fierer N, Lennon JT (2015) Resuscitation of the rare biosphere contributes to pulses of ecosystem activity. Front Microbiol 6:24. https://doi.org/10.3389/fmicb.2015.00024

Albarracin VH et al (2015) High-up: a remote reservoir of microbial extremophiles in Central Andean wetlands. Front Microbiol 6:1404. https://doi.org/10.3389/fmicb.2015.01404

Aszalós JM, Krett G, Anda D, Márialigeti K, Nagy B, Borsodi AK (2016) Diversity of extremophilic bacteria in the sediment of high-altitude lakes located in the mountain desert of Ojos del Salado volcano, Dry-Andes. Extremophiles 20:603-620. https:// doi.org/10.1007/s00792-016-0849-3

Battistuzzi FU, Hedges SB (2008) A major clade of prokaryotes with ancient adaptations to life on land. Mol Biol Evol 26:335-343

Blanco S et al (2013) New diatom taxa from high-altitude Andean saline lakes. Diatom Res 28:13-27

Bull AT (2011) Actinobacteria of the extremobiosphere. In: Horikoshi K (ed) Extremophiles handbook. Springer, Tokyo, pp 1203-1240. https://doi.org/10.1007/978-4-431-53898-1_58

Bull AT, Asenjo JA (2013) Microbiology of hyper-arid environments: recent insights from the Atacama Desert, Chile. Antonie van Leeuwenhoek 103:1173-1179

Bull AT, Asenjo JA, Goodfellow M, Gomez-Silva B (2016) The Atacama Desert: technical resources and the growing importance of novel microbial diversity. Annu Rev Microbiol 70:215-234. https://doi.org/10.1146/annurev-micro-102215-095236

Busarakam K, Bull AT, Trujillo ME, Riesco R, Sangal V, van Wezel GP, Goodfellow M (2016) Modestobacter caceresii sp. nov., novel actinobacteria with an insight into their adaptive mechanisms for survival in extreme hyper-arid Atacama Desert soils. Syst Appl Microbiol 39:243-251. https://doi.org/10.1016/j. syapm.2016.03.007

Cabrol NA et al (2009) The High-lakes project. J Geophys Res Biogeosci. https://doi.org/10.1029/2008JG000818

Caruso T, Chan Y, Lacap DC, Lau MC, McKay CP, Pointing SB (2011) Stochastic and deterministic processes interact in the assembly of desert microbial communities on a global scale. ISME J 5:14061413. https://doi.org/10.1038/ismej.2011.21
Cordero RR, Seckmeyer G, Damiani A, Riechelmann S, Rayas J, Labbe F, Laroze D (2014) The world's highest levels of surface UV. Photochem Photobiol Sci 13:70-81

Cordero RR et al (2016) The solar spectrum in the Atacama Desert. Sci Rep 6:22457. https://doi.org/10.1038/srep22457

Costello EK, Halloy SRP, Reed SC, Sowell P, Schmidt SK (2009) Fumarole-supported islands of biodiversity within a hyperarid, high-elevation landscape on Socompa Volcano, Puna de Atacama, Andes. Appl Environ Microbiol 75:735-747. https://doi. org/10.1128/Aem.01469-08

Crits-Christoph A et al (2013) Colonization patterns of soil microbial communities in the Atacama Desert. Microbiome 1:28. https:// doi.org/10.1186/2049-2618-1-28

Daly MJ et al (2004) Accumulation of Mn(II) in Deinococcus radiodurans facilitates gamma-radiation resistance. Science 306:1025-1028

Dorador C, Vila I, Remonsellez F, Imhoff JF, Witzel KP (2010) Unique clusters of Archaea in Salar de Huasco, an athalassohaline evaporitic basin of the Chilean Altiplano. FEMS Microbiol Ecol 73:291-302. https://doi. org/10.1111/j.1574-6941.2010.00891.x

Dorador C, Vila I, Witzel K-P, Imhoff JF (2013) Bacterial and archaeal diversity in high altitude wetlands of the Chilean Altiplano. Fund Appl Limnol/Arch Hydrobiol 182:135-159

Georgiou CD et al (2015) Evidence for photochemical production of reactive oxygen species in desert soils. Nat Commun 6:7100

Gtari M et al (2012) Contrasted resistance of stone-dwelling Geodermatophilaceae species to stresses known to give rise to reactive oxygen species. FEMS Microbiol Ecol 80:566-577

Horikoshi K, Antranikian G, Bull AT, Robb FT, Stetter KO (2011) Extremophiles handbook. Springer, Tokyo

Houston J (2006) Evaporation in the Atacama Desert: an empirical study of spatio-temporal variations and their causes. J Hydrol 330:402-412. https://doi.org/10.1016/j.jhydrol.2006.03.036

Husson O (2013) Redox potential (Eh) and pH as drivers of soil/plant/ microorganism systems: a transdisciplinary overview pointing to integrative opportunities for agronomy. Plant Soil 362:389-417

Idris H, Goodfellow M, Sanderson R, Asenjo JA, Bull AT (2017a) Actinobacterial rare biospheres and dark matter revealed in habitats of the Chilean Atacama Desert. Sci Rep 7:8373. https://doi. org/10.1038/s41598-017-08937-4

Idris H, Nouioui I, Asenjo JA, Bull AT, Goodfellow M (2017b) Lentzea chajnantorensis sp nov., an actinobacterium from a very high altitude Cerro Chajnantor gravel soil in northern Chile. Antonie van Leeuwenhoek 110:795-802. https://doi.org/10.1007/ s10482-017-0851-5

Kim SM, Park SW, Park ST, Kim YM (2011) Terrabacter carboxydivorans sp. nov., a carbon monoxide-oxidizing actinomycete. Int J Syst Evol Microbiol 61:482-486. https://doi.org/10.1099/ ijs.0.020826-0

Kuhlman KR et al (2005) Enumeration, isolation, and characterization of ultraviolet (UV-C) resistant bacteria from rock varnish in the Whipple Mountains, California. Icarus 174:585-595

Lalonde I, Constant P (2016) Identification of unknown carboxydovore bacteria dominant in deciduous forest soil via succession of bacterial communities, coxL genotypes, and carbon monoxide oxidation activity in soil microcosms. Appl Environ Microbiol 82:1324-1333

Lynch MD, Neufeld JD (2015) Ecology and exploration of the rare biosphere. Nat Rev Microbiol 13:217-229. https://doi.org/10.1038/ nrmicro3400

Lynch RC, King AJ, Farías ME, Sowell P, Vitry C, Schmidt SK (2012) The potential for microbial life in the highest-elevation (> 6000 m.a.s.l.) mineral soils of the Atacama region. J Geophys Res Biogeosci 117:G02028. https://doi.org/10.1029/2012jg001961 
Lynch RC, Darcy JL, Kane NC, Nemergut DR, Schmidt SK (2014) Metagenomic evidence for metabolism of trace atmospheric gases by high-elevation desert Actinobacteria. Front Microbiol 5:698. https://doi.org/10.3389/fmicb.2014.00698

Miyamoto KT, Komatsu M, Ikeda H (2014) Discovery of gene cluster for mycosporine-like amino acid biosynthesis from Actinomycetales microorganisms and production of a novel mycosporinelike amino acid by heterologous expression. Appl Environ Microbiol 80:5028-5036. https://doi.org/10.1128/AEM.00727-14

Molina V, Hernández K, Dorador C, Eissler Y, Hengst M, Pérez V, Harrod C (2016) Bacterial active community cycling in response to solar radiation and their influence on nutrient changes in a highaltitude wetland. Front Microbiol 7:1823. https://doi.org/10.3389/ fmicb.2016.01823

Navarro-Gonzalez R et al (2003) Mars-like soils in the Atacama Desert, Chile, and the dry limit of microbial life. Science 302:1018-1021. https://doi.org/10.1126/science.1089143

Norris PR (2012) Class II. Acidimicrobia class nov. In: Goodfellow M, Kämpfer P, Busse HJ, Trujillo ME, Suzuki KI, Ludwig W, Whitman W (eds) Bergey's manual of systematic bacteriology; the Actinobacteria, vol 5, 2nd edn. Springer, New York, p 1968

Oksanen J et al (2013) Package 'vegan'. Community ecology package for $\mathrm{R}$, version 241

Paulino-Lima IG, Fujishima K, Navarrete JU, Galante D, Rodrigues F, Azua-Bustos A, Rothschild LJ (2016) Extremely high UV-C radiation resistant microorganisms from desert environments with different manganese concentrations. J Photochem Photobiol B Biol 163:327-336

Rainey FA et al (2005) Extensive diversity of ionizing-radiation-resistant bacteria recovered from Sonoran Desert soil and description of nine new species of the genus Deinococcus obtained from a single soil sample. Appl Environ Microbiol 71:5225-5235

Schloss PD et al (2009) Introducing mothur: open-source, platformindependent, community-supported software for describing and comparing microbial communities. Appl Environ Microbiol 75:7537-7541. https://doi.org/10.1128/AEM.01541-09

Schmidt SK, Naff CS, Lynch RC (2012) Fungal communities at the edge: ecological lessons from high alpine fungi. Fungal Ecol 5:443-452

Sun H, Xu G, Zhan H, Chen H, Sun Z, Tian B, Hua Y (2010) Identification and evaluation of the role of the manganese efflux protein in Deinococcus radiodurans. BMC Microbiol 10:319

Ter Braak CJF (1986) Canonical correspondence analysis: a new eigenvector technique for multivariate direct gradient analysis. Ecology 67:1167-1179

Urzì C, Brusetti L, Salamone P, Sorlini C, Stackebrandt E, Daffonchio D (2001) Biodiversity of Geodermatophilaceae isolated from altered stones and monuments in the Mediterranean basin. Environ Microbiol 3:471-479

Wichner D et al (2017) Isolation and anti-HIV-1 integrase activity of lentzeosides AF from extremotolerant Lentzea sp. H45, a strain isolated from a high-altitude Atacama Desert soil. J Antibiot 70:448-453

Yoon SH, Ha SM, Kwon S, Lim J, Kim Y, Seo H, Chun J (2017) Introducing EzBioCloud: a taxonomically united database of 16S rRNA gene sequences and whole-genome assemblies. Int J Syst Evol Microbiol 67:1613-1617. https://doi.org/10.1099/ ijsem.0.001755

Zhou J et al (2002) Spatial and resource factors influencing high microbial diversity in soil. Appl Environ Microbiol 68:326-334 